\title{
Percepción de la imagen corporal como aproximación cualitativa
} al estado de nutrición*

Herlinda Madrigal-Fritsch, Lic. en Nutr., M.S.P., ${ }^{(1)}$ Jokin de Irala-Estévez, Ph. D., M.P.H., ${ }^{(2)}$ Miguel A. Martínez-González, Ph. D., M.P.H., ${ }^{(2)}$ John Kearney, Ph. D., ${ }^{(3)}$ Michael Gibney; Prof., M.D., ${ }^{(3)}$ J. Alfredo Martínez-Hernández, Prof., Ph. D. ${ }^{(4)}$

Madrigal-Fritsch H, de Irala-Estévez J,
Martínez-González MA, Kearney J, Gibney M,
Martínez-Hernández JA.
Percepción de la imagen corporal
como aproximación cualitativa
al estado de nutrición.
Salud Publica Mex I 999;4 I :479-486.

\section{Resumen}

Objetivo. Comparar la percepción de la imagen corporal con el índice de masa corporal (IMC) calculado a partir del peso y la talla declarados por los sujetos y valorar su capacidad para clasificar el estado de nutrición. Material y métodos. Se eligió una muestra representativa de la población de España, integrada por 517 hombres y 483 mujeres mayores de 15 años. Las variables fueron sexo, edad, nivel educativo, IMC e imagen corporal percibida. Las diferencias entre grupos se estimaron con la prueba de $\chi^{2}$. La capacidad de clasificación de la imagen corporal se comparó con el IMC empleando la sensibilidad y la especificidad. Resultados. El estado nutricional para ambos indicadores mostró mayor sobrepeso en hombres y mayor obesidad en mujeres. Se observó que el sobrepeso y la obesidad se incrementan conforme aumenta la edad, y con mayor educación disminuyen. La percepción de la imagen corporal fue distinta entre sexos, así como por edad y nivel educativo $(p<0.0 \mathrm{I})$. La comparación entre el IMC y la imagen corporal notificó valores superiores a 0.90 para sensibilidad y especificidad, así como para los valores predictivos positivos y negativos en sujetos que presentaron un estado nutricional que alcanzaba los rangos extremos. La precisión

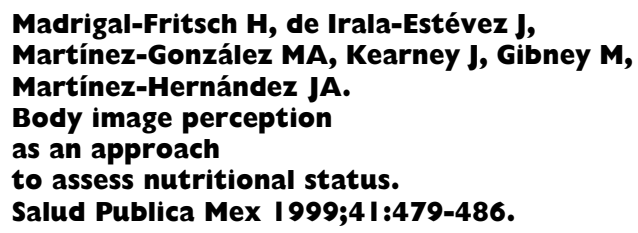

\section{Abstract}

Objective. To compare body image perception with body mass index (BMI) calculated from the weight and size declared by subjects, and to evaluate its usefulness in classifying the nutritional status. Material and methods. A representative sample of the Spanish population was selected consisting of 517 males and 483 females older than 15 years of age. Variables were sex, age, educational level, $\mathrm{BMI}$ and perceived body image. Differences between groups were estimated with the $\chi^{2}$ test. Specificity and sensitivity of the agreement between body mass and image were assessed. Results. The nutritional status for both indicators revealed higher overweight prevalence in males and obesity in females, which increase with age and decrease with greater educational level. Perceived body image differed between sexes, and among ages and educational levels $(p<0.0 \mathrm{I})$. Sensitivity and specificity as well as positive and negative predictive values were higher than 0.90 in subjects belonging to extreme cases. Precision was higher for sensitivity than for specificity. In all groups, categorization was better in females than in males and so was Spearman's correlation $(p<0.001)$. The Kendall W coefficient ranked high for both groups. Conclusions. Body image allowed the identifica-

* Proyecto financiado por European Commission, Directorate General V. Referencia: Agreement No. Soc 96201705 05F03 (96 CVVF3.429-0).

(I) Jefa del Departamento de Vigilancia Epidemiológica del Instituto Nacional de la Nutrición Salvador Zubirán, México, y profesora visitante del Departamento de Fisiología y Nutrición de la Universidad de Navarra, España.

(2) Departamento de Epidemiología y Salud Pública de la Universidad de Navarra, España.

(3) Instituto Europeo de Estudios de Alimentación de Dublín, Trinity College, Irlanda.

(4) Departamento de Fisiología y Nutrición de la Universidad de Navarra, España.

Fecha de recibido: II de marzo de 1999 - Fecha de aprobado: 2 de septiembre de 1999

Solicitud de sobretiros: Lic. Herlinda Madrigal. Departamento de Vigilancia Epidemiológica. Instituto Nacional de la Nutrición Dr. Salvador Zubirán. Vasco de Quiroga 15, Tlalpan, 14000 México, D.F., México.

Correo electrónico: hfritsch@quetzal.innsz.mx 
fue mayor para la sensibilidad que para la especificidad. La capacidad de clasificación fue mejor en mujeres que en hombres. La correlación de Spearman fue mayor en mujeres que en hombres $(p<0.001)$ y la concordancia $W$ de Kendall notificó valores altos para ambos sexos. Conclusiones. La percepción de la imagen corporal permitió identificar a los sujetos que presentaban nutrición normal y deficiente o excesiva; por tal motivo, este indicador puede resultar útil en estudios epidemiológicos, aunque tiene algunas limitaciones para diagnósticos individuales.

Palabras clave: imagen corporal; autoimagen; índice de masa corporal autodeclarado; estado nutricional; obesidad; España tion of individuals with normal, excessive or lean nutrition. This indicator can therefore be useful in epidemiological surveys, considering some limitations for individualized diagnoses.

Key words: body imagen; self concept; self-reported body mass index; nutritional status; obesity; Spain
$\mathrm{L}$ os especialistas en nutrición dedicados a la salud pública, siempre han manifestado especial interés por encontrar indicadores cuyo estudio implique un bajo costo y cuya aplicación en trabajos de campo se facilite, y que a su vez resulten aptos para identificar a las poblaciones en riesgo de mala nutrición, ya sea por déficit o por exceso de alimentación. ${ }^{1,2}$

En la última década ha aumentado la importancia de las enfermedades crónicas no transmisibles como problema prioritario de salud pública. Esta situación ha dejado de ser exclusiva de los países desarrollados y empieza a ser también motivo de preocupación en algunos países en vías de desarrollo. Esta situación se ha relacionado con cambios demográficos como el aumento en la esperanza de vida, la adopción de estilos de vida poco saludables y los acelerados procesos de urbanización. ${ }^{3-5}$ Las principales causas de mortalidad se han ido asemejando a las de los países industrializados (enfermedades cardiovasculares, tumores malignos, diabetes mellitus). Al mismo tiempo sigue existiendo una importante mortalidad por enfermedades transmisibles, propias de países en vías de desarrollo; a esta coexistencia, se le ha denominado transición epidemiológica., ${ }^{4,5}$

Ante este panorama, la investigación en los países en desarrollo se ha propuesto estimar la magnitud de los problemas de salud, identificar factores de riesgo a fin de establecer las medidas convenientes para su control. ${ }^{6-8}$ Los principales factores relacionados con la creciente presencia de enfermedades crónicas no transmisibles están asociados con patrones y hábitos de vida inadecuados, entre los que se pueden mencionar: el exceso en la alimentación en términos de energía, el desequilibrio en el aporte y el tipo tanto de grasas como de hidratos de carbono, así como la baja ingesta de fibra y de algunos micronutrientes. Otros importantes factores asociados al estilo de vida son el hábito tabáquico, el excesivo consumo de alcohol, la falta de ejercicio físico, el sedentarismo y el estrés, que favorecen diversos grados de sobrepeso y obesidad, y que contribuyen al desarrollo de otros padecimientos. ${ }^{9}$

Las encuestas nacionales que se han llevado a cabo en varios países durante los últimos años han incorporado diversos indicadores dietéticos, antropométricos y bioquímicos que permiten una mejor estimación de los problemas relacionados con la alimentación. ${ }^{10,11}$ Uno de los indicadores más empleados para medir el estado de nutrición en la población adulta es el índice de masa corporal (IMC), que se obtiene al dividir el peso en kilogramos entre la talla en metros elevada al cuadrado. ${ }^{12}$ La clasificación y los puntos de corte más aceptados en el ámbito internacional son: delgadez, que corresponde a un índice inferior a los $20 \mathrm{~kg} / \mathrm{m}^{2}$; normalidad, que oscila entre los 20 y los $24.99 \mathrm{~kg} / \mathrm{m}^{2}$; sobrepeso, que va de los 25 a los $29.99 \mathrm{~kg} / \mathrm{m}^{2}$, y obesidad, que alcanza los $30 \mathrm{~kg} / \mathrm{m}^{2}$ y más. ${ }^{13,14}$ Dicho índice habitualmente se obtiene en los estudios epidemiológicos, tanto haciendo las mediciones en los sujetos seleccionados, como registrando el peso y la talla que estos últimos declaran. Esta última técnica ha sido validada ampliamente y puede utilizarse sabiendo de antemano que subestima el peso y sobrestima la talla. ${ }^{15,16}$

En estudios realizados entre pacientes con trastornos del comportamiento alimentario, se emplea, asimismo, la imagen corporal real e ideal para captar el nivel de satisfacción que tienen respecto a su cuerpo, así como su grado de autoestima. ${ }^{17-22}$ También algunos autores han intentado validar la imagen corporal como un indicador de percepción del tamaño corporal. ${ }^{23-26}$

En el presente trabajo se pretende comparar la percepción de la imagen corporal con el IMC obtenido a partir del peso y la talla declarados por los sujetos que integraron la muestra española de la encuesta que 
el Instituto Europeo de Estudios en Alimentación (IEFS, por sus siglas en inglés), del Trinity College -ubicado en Dublín, Irlanda- llevó a cabo en 15 países de la Unión Europea (UE) durante 1997. ${ }^{27}$ Su objetivo fue estimar la capacidad de percepción de la propia imagen corporal para clasificar adecuadamente, en términos de prevalencia de sobrepeso y obesidad, de una muestra de sujetos adultos en un estudio epidemiológico.

\section{Material y métodos}

El estudio comprendió a 1000 sujetos mayores de 15 años (517 hombres y 483 mujeres) que conformaron la muestra española de la Encuesta Pan Europea, correspondiente al proyecto Body Weight and Physical Activity, 1997.28

Este proyecto incluyó a un total de 15239 sujetos en los 15 países miembros de la UE. El muestreo en cada país fue multietápico, estratificado según edad, sexo y distribución geográfica, y se aplicaron cuotas por sexo, edad y otras variables socioeconómicas para asegurar la representatividad. ${ }^{29}$

El cuestionario, diseñado por un grupo multidisciplinario de expertos y validado para tal efecto, contiene variables socioeconómicas, demográficas, antropométricas y algunas más relacionadas con actitudes y estilos de vida. La información se obtuvo mediante una entrevista directa llevada a cabo en los hogares y aplicada por profesionales específicamente entrenados, pertenecientes a la empresa de estudios de mercado Taylor-Nelson. ${ }^{29}$

Para el presente análisis se consideraron la imagen corporal percibida y el IMC, que se calculó con base en los datos declarados por los sujetos y dividiendo el peso en kilogramos, entre la talla en metros elevada al cuadrado. ${ }^{12}$

La información relacionada con la imagen corporal percibida se obtuvo pidiendo al sujeto entrevistado seleccionara la figura que, según su percepción, correspondía a su imagen corporal actual. Esta figura se debía elegir de entre nueve siluetas diferentes para hombres y mujeres (figura 1 ). ${ }^{29}$

La información se analizó por sexo, edad (en tres categorías: de 15 a 34 años, de 35 a 54 años y mayores de 55 años) y nivel de escolaridad (primario, secundario y terciario o universitario).

Con la imagen corporal percibida después de diversos análisis exploratorios -basados en la búsqueda de puntos de corte, con mejores valores de sensibilidad y especificidad que aseguraran buenos resultados para su aplicación en estudios epidemiológicos-, se establecieron cuatro categorías: delgado (silueta 1), normal

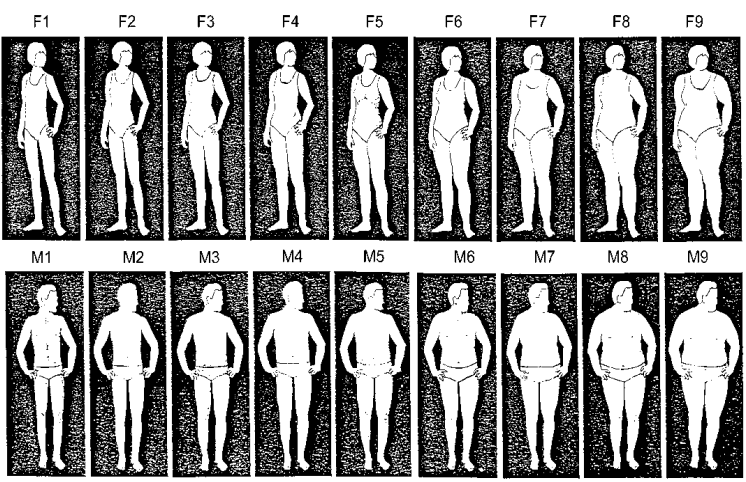

Figura I Opciones presentadas a cada encuestado PARA QUE SELECCIONASE LA SILUETA QUE MEJOR LE REPREsentaba. Muestra española de la Encuesta Pan EUROPEA, 1997

(siluetas 2-5), sobrepeso (siluetas 6 y 7) y obeso (siluetas 8 y 9), tanto para mujeres (F) como para varones $(\mathrm{M})$.

Para comparar la imagen corporal percibida con el IMC como referencia, se estimó la sensibilidad y la especificidad, así como los valores predictivos positivos y negativos, separadamente para hombres y mujeres; para estas estimaciones, se obtuvieron intervalos de confianza al $95 \%{ }^{30}$

Para obtener los valores de sensibilidad y especificidad se establecieron los siguientes puntos de corte entre la imagen corporal y el IMC: se consideró como delgados a aquellos sujetos que seleccionaron la silueta 1 y presentaron un IMC $<20 \mathrm{~kg} / \mathrm{m}^{2}$; como normales, a los que eligieron las siluetas 2 a 5 y presentaron un índice de $20-25 \mathrm{~kg} / \mathrm{m}^{2}$; con sobrepeso, a los que seleccionaron las siluetas 6 y 7 y tenían un IMC de 25-29 $\mathrm{kg} / \mathrm{m}^{2}$, y como obesos, a aquellos que eligieron las siluetas 8 y 9 y presentaron un índice 3230 .

También se calculó la correlación ordinal, por una parte, de las nueve figuras de la imagen corporal con el IMC como variable continua, mediante el coeficiente de correlación no paramétrico de Spearman, y, por la otra, de la imagen corporal con las cuatro categorías establecidas (delgadez, normalidad, sobrepeso, obesidad), mediante el coeficiente ordinal de concordancia W de Kendall.

\section{Resultados}

De la muestra total (517 hombres y 483 mujeres), el índice de respuesta alcanzó el $95 \%$ en las mujeres y el $96 \%$ en los hombres. 
La distribución por sexo del IMC y de la imagen corporal percibida, según los puntos de corte establecidos, permite observar que los hombres mostraron una mayor proporción de sobrepeso que las mujeres, mientras que respecto a la imagen corporal lo más destacable es que un mayor porcentaje de hombres se autocalifican en la normalidad (cuadro I).

$\mathrm{Al}$ analizar los dos indicadores por grupos de edad y sexo (cuadro II), se observa que la prevalencia de sobrepeso y obesidad aumenta con la edad en hombres y mujeres; asimismo al comparar ambos sexos, se encontraron diferencias estadísticamente significativas $(p<0.01)$.

La clasificación, en función de la edad, de la imagen corporal percibida (cuadro II) muestra tendencias y porcentajes similares a las del IMC tanto en hombres como en mujeres. También se encontraron diferencias estadísticamente significativas $(p<0.01)$ entre los grupos de edad para ambos sexos. $\mathrm{Al}$ comparar ambas distribuciones usando la imagen corporal percibida, se observó que la proporción de sujetos en los niveles de normalidad tendía a aumentar y que la prevalencia de sobrepeso en los hombres tendía a subestimarse, especialmente en el grupo de 15 a 34 años. En las mujeres lo más sobresaliente es que al usar la imagen corporal percibida se sobrestimaba el sobrepeso en el grupo de mayores de 55 años y que se subestimaba la delgadez en los grupos de 15 a 34 años y de 35 a 54, así como la obesidad en el grupo de mayores de 55 años.

$\mathrm{Al}$ estratificar por nivel educativo (cuadro III), se observó en ambos sexos que la mayor proporción se
Cuadro I

ESTADO NUTRICIONAL DE LA POBLACIÓN ESPAÑOLA MAYOR DE I 5 AÑOS DE EDAD, POR SEXO, SEGÚN ÍNDICE DE MASA CORPORAL E IMAGEN CORPORAL percibida. Encuesta Pan Europea, 1997

\begin{tabular}{cccccc} 
& \multicolumn{2}{c}{ Indice de masa corporal*a } & \multicolumn{2}{c}{ Imagen corporal } \\
\cline { 2 - 5 } \cline { 5 - 6 } Estado de nutrición & Hombres & Mujeres & Hombres & Mujeres \\
& $(n=505)$ & $(n=464)$ & $(n=5 / 4)$ & $(n=478)$ \\
& $\%$ & $\%$ & $\%$ & $\%$
\end{tabular}

\begin{tabular}{lrrrr} 
Delgadez & 3.4 & 14.0 & 5.8 & 4.0 \\
\hline Normalidad & 51.1 & 46.6 & 70.8 & 56.6 \\
\hline Sobrepeso & 35.8 & 27.8 & 19.6 & 31.0 \\
\hline Obesidad & 9.7 & 11.6 & 3.7 & 8.4 \\
Total & 100.0 & 100.0 & 100.0 & 100.0
\end{tabular}

* El índice de masa corporal fue calculado a partir del peso y la talla declarados por los sujetos

Nota: Comparación por sexo y por indicador: a) $\chi^{2}=39.1(p<0.001)$, b) $\chi^{2}=31.11(p<0.001)$

encontraba en el nivel de normalidad, y que a mayor nivel de educación existía menor prevalencia de sobrepeso y obesidad; cabe aclarar que esta tendencia fue mayor en las mujeres que en los hombres. Con la imagen corporal percibida se observó el mismo fenómeno que con el IMC, ya que a medida que aumentaba el nivel de educación disminuía la prevalencia de sobrepeso y obesidad. Sin embargo, nuevamente en el caso de los hombres se observó una marcada subes-

Cuadro II

ESTADO NUTRICIONAL DE LA POBLACIÓN ESPAÑOLA MAYOR DE I 5 AÑOS POR SEXO Y GRUPOS DE EDAD, según índice de masa corporal e imagen corporal percibida. Encuesta Pan Europea, I 997

\begin{tabular}{|c|c|c|c|c|c|c|c|c|c|c|c|c|}
\hline \multirow{4}{*}{$\begin{array}{l}\text { Estado de } \\
\text { nutrición }\end{array}$} & \multicolumn{6}{|c|}{ Indice de masa corporal* (Grupos de edad en años) } & \multicolumn{6}{|c|}{ Imagen corporal (Grupos de edad en años) } \\
\hline & & Hombres $^{\circ}$ & & & Mujeres ${ }^{b}$ & & & Hombresc & & & Mujeres ${ }^{d}$ & \\
\hline & $\begin{array}{c}15-34 \\
(n=231)\end{array}$ & $\begin{array}{c}35-54 \\
(n=122)\end{array}$ & $\begin{array}{l}55 \text { y más } \\
(n=152)\end{array}$ & $\begin{array}{l}15-34 \\
(n=182)\end{array}$ & $\begin{array}{c}35-54 \\
(n=150)\end{array}$ & $\begin{array}{l}55 \text { y más } \\
(n=132)\end{array}$ & $\begin{array}{c}15-34 \\
(n=235)\end{array}$ & $\begin{array}{c}35-54 \\
(n=123)\end{array}$ & $\begin{array}{l}55 \text { y más } \\
(n=156)\end{array}$ & $\begin{array}{l}15-34 \\
(n=185)\end{array}$ & $\begin{array}{c}35-54 \\
(n=152)\end{array}$ & $\begin{array}{l}55 \text { y más } \\
(n=\mid 4 I)\end{array}$ \\
\hline & $\%$ & $\%$ & $\%$ & $\%$ & $\%$ & $\%$ & $\%$ & $\%$ & $\%$ & $\%$ & $\%$ & $\%$ \\
\hline Delgadez & 4.8 & 2.5 & 2.0 & 26.9 & 7.3 & 3.8 & 6.8 & 5.7 & 4.5 & 7.0 & 1.3 & 2.8 \\
\hline Normalidad & 66.2 & 43.4 & 34.2 & 54.4 & 46.1 & 36.4 & 83.9 & 67.5 & 53.8 & 74.1 & 56.6 & 34.0 \\
\hline Sobre peso & 25.1 & 45.1 & 44.7 & 13.2 & 35.3 & 39.4 & 8.9 & 21.9 & 34.0 & 15.7 & 33.6 & 48.3 \\
\hline Obesidad & 3.9 & 9.0 & 19.1 & 5.5 & 11.3 & 20.4 & 0.4 & 4.9 & 7.7 & 3.2 & 8.5 & 14.9 \\
\hline Total & 100.0 & 100.0 & 100.0 & 100.0 & 100.0 & 100.0 & 100.0 & 100.0 & 100.0 & 100.0 & 100.0 & 100.0 \\
\hline
\end{tabular}


timación del sobrepeso y la obesidad, de tal forma que se encontraron diferencias estadísticamente significativas entre los sexos para ambos indicadores $(p<0.01)$.

Los resultados de la sensibilidad, la especificidad y los valores predictivos del indicador de imagen corporal con referencia al IMC (cuadro IV) permiten observar que la aproximación cualitativa de la imagen es capaz de identificar a los sujetos que se encuentran en los grupos extremos: sobrepeso y obesidad respecto a delgadez, con valores de sensibilidad y especificidad superiores a 0.90. La precisión de dichas estimaciones fue alta en el caso de la sensibilidad y algo menor para la especificidad, de tal modo que se encontraron precisiones comparables para los valores predictivos positivos y negativos. En el caso de las comparaciones entre los grupos con estado de nutrición deficitario o con excesivo sobrepeso respecto a la normalidad, sólo para la especificidad y los valores predictivos negativos se encontraron cifras por encima de 0.80 . En cambio, la sensibilidad para distinguir la delgadez y el sobrepeso frente a la normalidad, y la obesidad frente al sobrepeso fue inaceptablemente baja; también lo

\section{Cuadro III

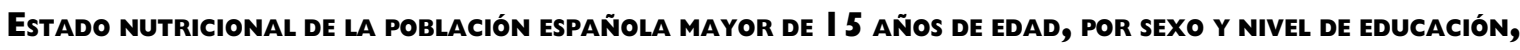 según índice de masa corporal e imagen corporal percibida. Encuesta Pan Europea, 1997}

\begin{tabular}{|c|c|c|c|c|c|c|c|c|c|c|c|c|}
\hline \multirow{4}{*}{$\begin{array}{l}\text { Estado de } \\
\text { nutrición }\end{array}$} & \multicolumn{6}{|c|}{ Índice de masa corporal* (Nivel de educación) } & \multicolumn{6}{|c|}{ Imagen corporal (Nivel de educación) } \\
\hline & \multicolumn{3}{|c|}{ Hombres $^{a}$} & \multicolumn{3}{|c|}{ Mujeres $^{b}$} & \multicolumn{3}{|c|}{ Hombres $^{c}$} & \multicolumn{3}{|c|}{ Mujeres $^{d}$} \\
\hline & $\begin{array}{l}\text { Primario } \\
(n=319)\end{array}$ & $\begin{array}{c}\text { Secundario } \\
(n=122)\end{array}$ & $\begin{array}{l}\text { Terciario } \\
(n=64)\end{array}$ & $\begin{array}{l}\text { Primario } \\
(n=288)\end{array}$ & $\begin{array}{l}\text { Secundario } \\
(n=12 I)\end{array}$ & $\begin{array}{l}\text { Terciario } \\
(n=55)\end{array}$ & $\begin{array}{l}\text { Primario } \\
(n=325)\end{array}$ & $\begin{array}{l}\text { Secundario } \\
(n=123)\end{array}$ & $\begin{array}{l}\text { Terciario } \\
(n=66)\end{array}$ & $\begin{array}{l}\text { Primario } \\
(n=298)\end{array}$ & $\begin{array}{l}\text { Secundario } \\
(n=123)\end{array}$ & $\begin{array}{l}\text { Terciario } \\
(n=57)\end{array}$ \\
\hline & $\%$ & $\%$ & $\%$ & $\%$ & $\%$ & $\%$ & $\%$ & $\%$ & $\%$ & $\%$ & $\%$ & $\%$ \\
\hline Delgadez & 2.8 & 3.3 & 6.2 & 9.0 & 24.0 & 18.2 & 5.2 & 7.3 & 6.1 & 4.0 & 4.0 & 3.5 \\
\hline Normalidad & 44.2 & 68.2 & 53.2 & 38.9 & 56.2 & 65.4 & 64.9 & 80.5 & 81.8 & 45.3 & 74.0 & 79.0 \\
\hline Sobrepeso & 39.8 & 25.4 & 35.9 & 36.1 & 14.8 & 12.7 & 24.3 & 12.2 & 10.6 & 39.6 & 17.9 & 14.0 \\
\hline Obesidad & 13.2 & 3.3 & 4.7 & 16.0 & 5.0 & 3.7 & 5.6 & 0.0 & 1.5 & II.I & 4.0 & 3.5 \\
\hline Total & 100.0 & 100.0 & 100.0 & 100.0 & 100.0 & 100.0 & 100.0 & 100.0 & 100.0 & 100.0 & 100.0 & 100.0 \\
\hline
\end{tabular}

* El índice de masa corporal fue calculado a partir del peso y la talla declarados por los sujetos

Nota: comparación por educación, sexo y por indicador: a) $\chi^{2}=27.6(p<0.01)$, b) $\chi^{2}=55.8(p<0.01)$, c) $\chi^{2}=23.2(p<0.01)$, d) $\chi^{2}=44.5(p<0.01)$

\section{Cuadro IV}

SENSIBILIDAD, ESPECIFICIDAD E INTERVALOS DE CONFIANZA Y VALORES PREDICTIVOS POSITIVOS Y NEGATIVOS DEL ESTADO NUTRICIONAL DE LA POBLACIÓN ESPAÑOLA MAYOR DE I 5 AÑOS DE EDAD, POR SEXO, según índice de masa corporal* e imagen corporal percibida. Encuesta Pan Europea, 1997

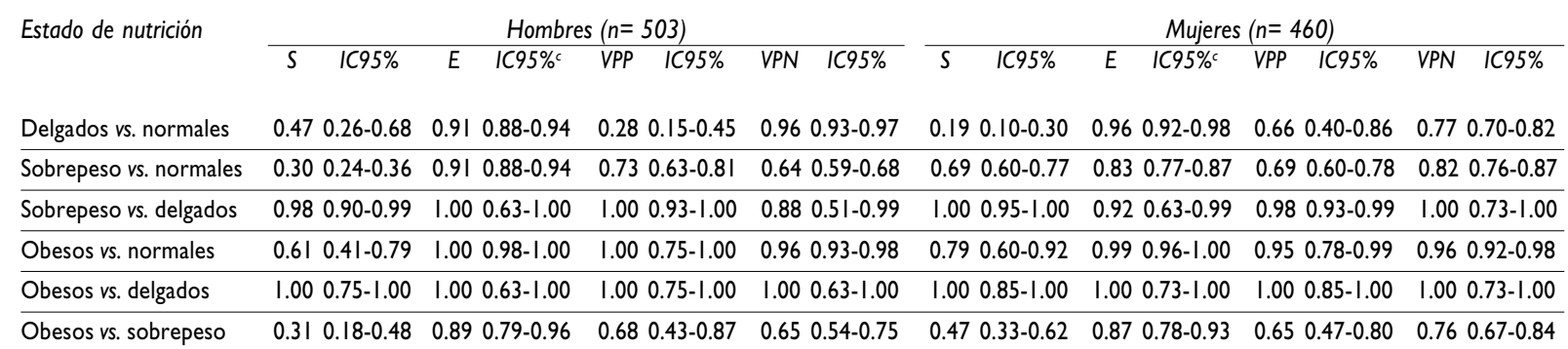

* El índice de masa corporal fue calculado a partir del peso y la talla declarados por los sujetos

S: sensibilidad

E: especificidad

IC: intervalo de confianza
VPP: valor predictivo positivo

VPN: valor predictivo negativo 
fueron los valores predictivos positivos. En general, fue más fácil clasificar a las mujeres que a los hombres, a partir de la percepción de la imagen corporal.

El coeficiente de correlación de Spearman por sexo entre ambos indicadores, comparando las nueve figuras de la imagen corporal con los valores categorizados del IMC, reveló una alta asociación $(p<0.001)$ con un coeficiente $(r)$ mayor en mujeres (0.69) que en hombres (0.64). Además, existía una concordancia significativamente alta entre los dos indicadores (imagen corporal $v s$. IMC), la que se expresó más claramente en mujeres que en hombres ( $\mathrm{W}$ de Kendall: 0.87 y 0.58 , respectivamente).

\section{Discusión}

La necesidad de realizar estudios epidemiológicos en grandes muestras de población y, al mismo tiempo, optimizar recursos reduciendo el costo, el tiempo y el esfuerzo que supone la ejecución del trabajo de campo, sin perder la calidad de la información, ha conducido a los investigadores a buscar métodos simplificados para llevarlos a cabo. Un ejemplo de ello se tiene en un estudio clásico de epidemiología sobre hábito tabáquico y cáncer de pulmón, ${ }^{31}$ en el cual la información se obtuvo mediante una encuesta enviada por correo y autocontestada; asimismo, en epidemiología nutricional existen varios estudios de evaluación de ingesta de alimentos hechos con cuestionarios enviados por correo autocontestados o con encuestas realizadas por teléfono. ${ }^{32}$ En estudios de valoración del estado de nutrición en población adulta se han aplicado otras técnicas para obtener el peso y la talla, sin necesidad de hacer la medición directa del sujeto estudiado. ${ }^{33-35}$ En algunos de estos estudios, los sujetos seleccionados refieren su peso y su talla, o bien, ellos mismos realizan la medición de perímetros corporales, ya que se suele anexar una cinta métrica al cuestionario. ${ }^{36}$

Todas estas experiencias se han realizado en poblaciones que presentan determinadas características socioeconómicas y culturales como son: alto nivel de instrucción (todos los sujetos seleccionados han sido alfabetas y capaces de contestar un cuestionario siguiendo instrucciones escritas o dadas por teléfono), buen grado de concienciación (han aceptado participar en el estudio, pensando en los beneficios para la salud de la población) y con adecuado acceso a la información y a los servicios de salud.

Los investigadores que han diseñado estos métodos alternativos, los recomiendan como un modo eficiente de realizar estudios similares, una vez que han hecho la validación correspondiente, es decir, cuando han identificado la exactitud (que el instrumento sea capaz de obtener la información que realmente se desea obtener) y la replicabilidad (que proporcione la misma información cuando se aplique repetidamente en las mismas muestras). Estos autores han debido comparar el método o el indicador que desean validar, con el "patrón de oro", y realizar el estudio en muestras representativas de la población. ${ }^{2}$

Por todo lo anterior, en este trabajo se pretendió estimar la capacidad de clasificar a la población en las categorías relevantes desde el punto de vista antropométrico, utilizando la imagen corporal percibida y comparándola con el índice de masa corporal autodeclarado; además, cabe recordar que se utilizó la información de una encuesta llevada a cabo en una muestra representativa de la población española. Por último, es preciso señalar que el IMC autodeclarado, como indicador del estado de nutrición de la población adulta, ya se ha utilizado ampliamente en estudios epidemiológicos bien conocidos. ${ }^{37,38}$

La información obtenida en este trabajo muestra que la clasificación que se estableció para la imagen corporal percibida, permitió identificar a la población que se encontraba en los grados extremos del estado de nutrición (delgados, con sobrepeso y obesos), ya que los valores de sensibilidad y especificidad obtenidos fueron altos. Asimismo, permitió identificar a aquellos que se encontraban en condiciones de normalidad, con valores altos de especificidad. Las pruebas de correlación de Spearman y la de concordancia de Kendall mostraron buenos valores, pero fueron siempre superiores en las mujeres que en los hombres. En cambio, la sensibilidad y el valor predictivo positivo en algunas comparaciones fueron bajas, especialmente entre los varones. Por tanto, la capacidad del método cualitativo de la imagen corporal para una discriminación más fina entre categorías adyacentes es limitada y tiende a subestimar la prevalencia, tanto de sobrepeso como de obesidad.

El conjunto de resultados obtenidos al estratificar por diferentes variables (sexo, edad y educación), así como la comparación entre imagen percibida e IMC calculado a partir de lo que los sujetos declaran, señalan una tendencia semejante a las encontradas en otras poblaciones cuando se han utilizado métodos que consideran la medición directa del peso y de la altura. ${ }^{14}$ Así, ambos procedimientos indican que la prevalencia de obesidad es mayor en la mujer que en el varón, aumenta con la edad y disminuye con el nivel de educación. En cualquier caso, es posible que el varón esté menos preocupado por su imagen $y$, por ello, tienda a 
subestimar su sobrepeso/obesidad con mayor frecuencia que la mujer, quien probablemente sea más consciente de su imagen corporal.

Estos primeros hallazgos llevan a proponer un futuro estudio de validación, donde se compare la imagen corporal percibida con el índice de masa corporal ya no autodeclarado, sino directamente medido en muestras representativas de la población, que presenten condiciones socioeconómicas distintas a las referidas anteriormente. También resultaría interesante comprobar si la percepción de la imagen corporal reduce el sesgo asociado al hecho de que el IMC sea autodeclarado y si permite una mejor clasificación de los sujetos. Además, habría que realizar los estudios de validación en poblaciones analfabetas, sin acceso a servicios de salud y con menor información y responsabilidad sobre su propia salud.

La validación de este indicador será de gran utilidad, ya que además de disminuir sensiblemente el costo y el tiempo de ejecución de los estudios, podría aplicarse rápida y fácilmente en aquellos estudios epidemiológicos que incluyan población en la que se dificulte realizar las mediciones, como es el caso de las personas de la tercera edad o, en México y Latinoaméri$\mathrm{ca}$, poblaciones localizadas en zonas rurales muy dispersas y de difícil acceso. Estos resultados sugieren que la mujer -habitualmente la persona más entrevistada en encuestas nacionales- podría referir con más objetividad los datos relacionados con su peso, estatura e imagen corporal; si ella también hiciera referencia a los datos de su pareja, muy probablemente se reduciría la diferencia respecto a la percepción encontrada en estudios donde cada sexo autodeclara sus datos.

\section{Agradecimientos}

Se reconoce con gratitud la colaboración científica y financiera del Instituto Europeo de Estudios de Alimentación (IEFS, Dublín, Irlanda) y al Directorato General $\mathrm{V}$ de la Unión Europea

\section{Referencias}

I. Stundkard AJ, Albaum JM. The accuracy of self reported weights. Am J Clin Nutr 1981; 34:1593 - 1599.

2.. Hernández M, Romieu I, Parra S, Hernández J, Madrigal H, Willet W. Validity and reproducibility of a food frequency questionnaire to assess dietary intake of women living in Mexico City. Salud Publica Mex 1998; 39:133-140.
3. Frenk J, Frejka T, Bobadilla JL, Stern C, Lozano R, Sepúlveda J et al. La transición epidemiológica en América Latina. Bol Oficina Sanit Panam 1991; I I :485-496.

4. Albala C, Vio F. Epidemiological transition in Latin America; The case of Chile. Public Health 1995; 109:431-442.

5. Albala C, Vio F, Yáñez M. Transición epidemiológica en América Latina: comparación de cuatro países. Rev Med Chil 1997;125:719-727.

6. Day EN, Varghese C. Oesophageal cancer. Cancer Surv 1994;19:43-54.

7. The INCLEN Multicentre Collaborative Group. Socioeconomic status and risk for cardiovascular disease: A multicentre collaborative study in the International Clinical Epidemiology Network (INCLEN). J Clin Epidemiol 1994;47:140I-I409.

8. Murray CJ, Lopez AD. Global mortality, disability and contribution of risk factors: Global Burden of Disease Study. Lancet 1997;349: I 436-1442. 9. Martorell R, Khan LK, Hughes ML, Grummer LM. Obesity in Latin American women and children. J Nutr 1998; 128: 1464-1473.

10. Sepúlveda J, Lezana MA, Tapia R, Valdespino JL, Madrigal H, Kumate J. Estado nutricional de preescolares y mujeres en México: resultados de una encuesta probabilística nacional. Gac Med Mex 1990;126:207-225.

II. Vital and health statistics. Dietary methodology workshop for the Third National Health and Nutrition Examination Survey. Hyattsville: US Department of Health and Human Services, 1992;DHHS Publication N. (PHS) 92-1964.

12. Quetelet LA. Physique Sociale: OU, essai sur le develloppement des facultes de' homme. Brussels, Belgium: C, Muquardt, 1969.

13. Bray GA, Bouchard C, James WP. Definitions and proposed current classification of obesity in handbook of obesity. Nueva York: Marcel Dekker, 1997:31-32.

14. World Health Organization. Obesity preventive and managing the global epidemiol. Report of a WHO consultation on obesity; 1997 june 3-5; Geneve. Ginebra: Division of Noncomunicable Disease, Programme of Nutrition Family and Reproductive Health; (WHO/NUT/NCD/98.I), 1998:276.

15. Stewart AL. The reliability and validity of self-reported weight and height. J Chronic Dis 1982;35:295-309.

16. Stewart AW, Jackson MA, Ford MA, Beaglehole R. Underestimation of relative weight by use of self -reported height and weight. Am J Epidemiol 1987;125:122-126.

17. Thompson JK, Fabian LJ, Moulton DO, Dunn ME, Altabe MN. Development and validation of physical appearance related teasing scale. J Pers Assess 1991;56:513-521.

18. Sullivan M, Karlsson J, Sjostrom L, Backman L, Bengtsson C, Bouchard $C$ et al. Swedish obese subjects (SOS): An intervention study of obesity. Baseline evaluation of health and psychosocial functioning in the first 1743 subjects examined. Int J Obes Relat Metab Disord 1993; 17:503-5 I2.

19. Davis C, Durnin JV, Dionne M, Gurevich M. The influence of body fat content and bone diameter measurements on body dissatisfaction in adult women. Int J Eat Disord 1994;15: 257-263.

20. Stevens J, Kumaylka SH, Keil J. Attitudes toward body size and dieting. Differences between elderly black and white women. Am J Public Health 1994;84:1322-1325.

21. Gittelsohn J, Harris SB, Lyman TH, Hanley A, Barnie A., Zinman B. Body image concepts differ by age and sex in an Ojibway-Cree Community in Canada. J Nutr 1996; 126:2990-3000.

22. Grilo CM, Wilfley DE, Brownell KD, Rodin J. Teasing, body image, and self-esteem in a clinical sample of obese women. Addict Behav 1994;19: 443-450.

23. Thompson MA, Gray JJ. Development and validation of a new body image assessment scale. J Pers Assess 1995;64:258-269.

24. Jeffery RW. Bias in reported body weight as a function of education, occupation, health and weight concern. Addict Behav 1996;21:217-222.

25. Sisson BA, Franco SM, Carlin WM, Mitchell CK. Body fat analysis and perception of body image. Clin Pediatr (Phila) 1997;36:415-418. 
26. Cachelin FM, Striegel RH, Elder KA. Realistic weight perception and body size assessment in a racially diverse community sample of dieters. Obes Res 1998;6:62-68.

27. Strain JJ. A Pan EU Survey of Consumer Attitudes to food, nutrition and health. Eur J Clin Nutr 1997;51:I-58.

28. Martínez JA, Kearney JM, Kafatos K, Paquet S, Martínez-González MA. Variables independently associated with self-reported obesity (BMI $>30 \mathrm{~kg} / \mathrm{m}^{2}$ ) in the European Union population. Public Health Nutr 1999;2:125-133.

29. Kearney M, Kearney JM, Gibney MJ. Methods used to conduct the survey on consumer attitudes to food, nutrition and health on nationally representative samples of adults from each member state of the European Union. Eur J Clin Nutr 1997;51:3-7.

30. Nieto FJ, Bush TL, Keyl PM. Body mass definitions of obesity: Sensitivity and specificity using self-reported weigth and height. Epidemiology 1990;: 1:146-152.

3I. Doll R, Hill AB. Lung cancer and other causes of death in relation to smoking. A second report on the mortality of British doctors. BMJ 1956;2:1071.

32. Serra LL. Evaluación del consumo de alimentos en poblaciones. Encuestas alimentarias. En: Serra LL, Aranceta J, Mataix J. Nutrición y salud pública. Métodos, bases científicas y aplicaciones. Barcelona: Editorial Masson, 1995; I:40I.

33. Palta M, Prineas RJ, Berman R, Hanan P. Comparison of self-reported and measured height and weight. Am J Epidemiol I982; I 15:223-230.

34. Rimm EB, Stampfer MJ, Colditz GA, Chute CG, Litin LB, Willet W. Validity of self-reported waist and hip circunferences in men and women. Epidemiology 1990; I:466-473.

35. Stevens J, Keil JE, Waid LR, Gazes PC. Accuracy of current, 4 -year, and 28- year self-reported body weight in an elderly population. Am J Epidemiol 1990;132:1156-1163.

36. Willet W. Anthropometric measures and body composition. En: Willet W. Nutrition epidemiology. 2a. edición. Nueva York: Oxford University Press, 1998:514.

37. Faculty of Health Medicine and Biological Sciencies, University of Southampton. GP documentation of obesity: What does it acchieve? Br J Gen Pract 1998:48:890-894.

38. Giacchi M, Mattei R, Rossi S. Correction of the self-reported BMI in a teenage population. Int J Obes Relat Metab Disord 1998;22:673-677. 